\title{
Evaluation of a peer led parenting intervention for disruptive behaviour problems in children: community based randomised controlled trial
}

\author{
(C) $(1)(9)$ OPEN ACCESS
}

\author{
Crispin Day head ${ }^{12}$, Daniel Michelson senior clinical research associate ${ }^{1}$, Stacey Thomson \\ postdoctoral researcher ${ }^{1}$, Caroline Penney specialist trainer ${ }^{2}$, Lucy Draper specialist trainer ${ }^{2}$
}

${ }^{1}$ Child and Adolescent Mental Health Services Research Unit, Department of Psychology, Institute of Psychiatry, King's College London, London SE5 8AF, UK; ${ }^{2}$ Centre for Parent and Child Support, South London and Maudsley NHS Foundation Trust, Michael Rutter Centre, London

\begin{abstract}
Objective To evaluate the effectiveness of a peer led parenting intervention delivered to socially disadvantaged families.

Design Randomised controlled trial.

Setting Schools and children's centres in a socially deprived borough of inner London.

Participants Parental caregivers seeking help with managing the problem behaviours of 116 index children, aged 2-11 years; 59 families were randomised to the intervention and 57 to a waitlist control condition.

Intervention Empowering parents, empowering communities is an eight week (two hours each week), manualised programme delivered to groups of parents by trained peer facilitators from the local community.

Main outcome measures Child problems (number and severity), parental stress, and parenting competencies were assessed before and after the intervention using standardised parent reported measures.

Results Significantly greater improvements in positive parenting practices and child problems were observed in the intervention group compared with the waitlist group, with no difference in parental stress between the groups. An intention to treat analysis for the primary outcome measure, the intensity subscale of the Eyberg child behaviour inventory, showed an intervention effect size of 0.38 (95\% confidence interval 0.01 to 0.75 , $\mathrm{P}=0.01$ ). The intervention group had high rates of treatment retention $(91.5 \%)$ and user satisfaction.
\end{abstract}

Conclusion The peer led parenting intervention significantly reduced child behaviour problems and improved parenting competencies. This is a promising method for providing effective and acceptable parenting support to families considered hard to reach by mainstream services.

Trial registration Current Controlled Trials ISRCTN01962337.

\section{Introduction}

Effective parenting, characterised by consistent, supportive, and responsive childrearing practices, is critical to achieving positive developmental outcomes for children. ${ }^{1}$ Links between parenting styles and the causation, maintenance, and amelioration of mental health difficulties in young people, and disruptive behaviour in particular, have also been well established. ${ }^{2}$ Disruptive behaviour, which includes aggression, non-compliance, and oppositionality, is the most common reason for referral to child mental health clinics in the United Kingdom and other developed countries, with the highest prevalence in inner city areas. ${ }^{34}$ These challenging behaviours impact considerably on quality of life for the affected children and their families and are closely linked to academic failure, crime, unemployment, and other adverse long term outcomes, with high social and economic costs. ${ }^{5}$ The provision of effective and accessible treatment programmes is therefore of major national and international importance.

Numerous clinical trials have shown that parenting interventions developed from social learning theory and relationship based approaches improve disruptive behaviour problems in children. ${ }^{6}$ These interventions are consequently recommended for routine practice by the National Institute for Health and Clinical Excellence ${ }^{7}$ (an independent body that provides national guidance on health interventions for England and Wales). However, many of the families who are most in need of treatment do not access appropriate care, either dropping out prematurely or failing to engage with services from the outset. ${ }^{8}$ This may be due in part to logistical barriers, such as competing demands and difficulties with transportation, which are especially salient for highly stressed and isolated families living in areas of socioeconomic disadvantage. ${ }^{9}$ Negative parental expectancies about treatment, including concerns about the cultural acceptability of conventional parenting interventions, may also hinder initial and subsequent engagement. ${ }^{10}$

The empowering parents, empowering communities programme was developed with such hard to reach families in mind. 
Drawing on a peer led service delivery model that has been successfully applied to several other health conditions and underserved populations, ${ }^{12} 1314$ the programme trains local parents as peer facilitators to deliver a manualised, group based parenting intervention in community settings. As well as showing high rates of user satisfaction, an uncontrolled pilot evaluation ${ }^{15}$ found that attendance at the peer led parenting groups was associated with significant reductions in parent reported child behaviour problems.

We evaluated the clinical effectiveness and acceptability of the programme using a more rigorous randomised controlled trial methodology, applied to a population of families living in an economically deprived, inner city area. We tested the hypotheses that participation in the peer led parenting intervention would be associated with significant advantages over a waitlist control condition, in terms of improved child behaviour problems, increased use of positive parenting practices, and reduced parental stress.

\section{Methods}

This multisite randomised controlled trial was designed in accordance with the consolidated standards of reporting trials guidelines. ${ }^{16} \mathrm{We}$ compared an intervention group with a waitlist control condition, using a 1:1 allocation ratio. Informed consent was obtained from all participants before data collection and randomisation.

\section{Study population}

The trial took place between January and December 2010 at six sites (three schools, two children's centres/schools, and one church) across Southwark. This inner London borough is one of the most deprived local authorities in England, with a high proportion of residents from black and ethnic minority groups and more than double the national average rate of severe emotional and behavioural difficulties among youths. ${ }^{4}$ Recruitment started immediately after ethical approval was obtained, and the trial was registered with Current Controlled Trials two weeks later owing to administrative delays.

Families were eligible for inclusion if a primary parental caregiver ("parent") had identified difficulties in managing the behaviour of an index child aged between 2 and 11 years, in the absence of serious neurodevelopmental problems. We excluded families from the study if the parent was unable to read and write in English, could not commit to weekly attendance at the parenting groups, and was not currently living at home with the index child. To increase accessibility of the programme, we set no specific inclusion or exclusion criteria for the type or severity of child problems, other than the fact that the parent was seeking help with managing difficult behaviours. In households with more than one child aged 2-11 years, we asked the parents to nominate an index child whose behaviour was of greatest concern.

Families were recruited through word of mouth, posters in schools and children's centres, professional referrals from social workers and schools, and face to face contacts by programme outreach workers. The families of 116 index children were eligible and consented to take part; 59 families were randomised to the intervention group and 57 to the waitlist control group. For five families both parents participated in the trial; in these instances we collected data from one self nominated parent (usually the mother). In all the other families, only one parent took part. Fifty four families were available for follow-up in the intervention group, and 50 were retained in the waitlist control group. The 12 families that were lost to follow-up were included in an intention to treat analysis.

\section{The intervention}

Pairs of trained peer facilitators delivered the programme (empowering parents, empowering communities) to groups of 7-14 parents over the course of eight weekly, two hour sessions. The parenting groups aimed to improve parent-child relationships and interactions, reduce behavioural problems in the child, and increase participants' confidence in their parenting abilities. The course was delivered according to a structured manual ${ }^{17}$ and employed attachment, social learning, structural, relational, and cognitive behavioural theories and methods, consistent with national guidelines ${ }^{7}$ for the treatment of disruptive behaviour in children (box). Intervention sessions involved sharing of information, group discussion, demonstration, role play, reflection, and planning/review of homework tasks.

A cohort of 12 peer facilitators was responsible for delivering six parenting groups in the pre-crossover phase of the trial. These peer facilitators were themselves parents from the local community who had successfully completed an accredited training programme that included workshops (totalling 60 hours), submission of a written portfolio, and a period of supervised practice. ${ }^{15}$ Group facilitators received fortnightly supervision to enhance intervention fidelity, skill development, and personal support. Supervisors were also contactable by telephone to manage potential safeguarding issues - for example, recognition and reporting of abusive parenting practices.. The direct costs of running the programme (including facilitators' wages, travel expenses, printing of materials, accreditation, crèche workers, refreshments, and rental of a room) were about $£ 2700$ ( $€ 3249$; \$4273) per group.

\section{Outcome measures}

We assessed the outcomes for children and parents using a set of standardised, parent reported questionnaires, all of which had been extensively piloted with the target population and widely used in other parent training trials. The measures were administered to groups of parents at study sites one week before the start of the intervention and immediately after the final intervention session. We individually followed-up parents who were unable to attend the group data collection sessions.

\section{Primary outcome measure}

The Eyberg child behaviour inventory ${ }^{18}$ is a 36 item scale that assesses both the number of child disruptive behaviour problems (problems subscale) and the frequency with which these occur (intensity subscale). We selected the intensity subscale as the primary outcome for the study, given its excellent sensitivity $(96 \%)$ and specificity $(87 \%)^{19}$ for detecting disruptive behaviour (relative to the structured interview for disruptive behaviour disorders of the Diagnostic and Statistical Manual of Mental Disorders, third edition, revised, DSM-III-R) and frequent use in previous trials of parenting interventions for disruptive behaviour in children. ${ }^{6}$ The measure also has acceptable inter-rater reliability $(\kappa 0.61$ to 0.79$){ }^{20}$

\section{Secondary outcome measures}

The concerns about my child ${ }^{21}$ measure is a visual analogue scale that requires parents to nominate, prioritise, and rate up to three key concerns about their child. The same concerns that were nominated at baseline were re-rated at follow-up, providing a sensitive, individualised index of change. Given expected 


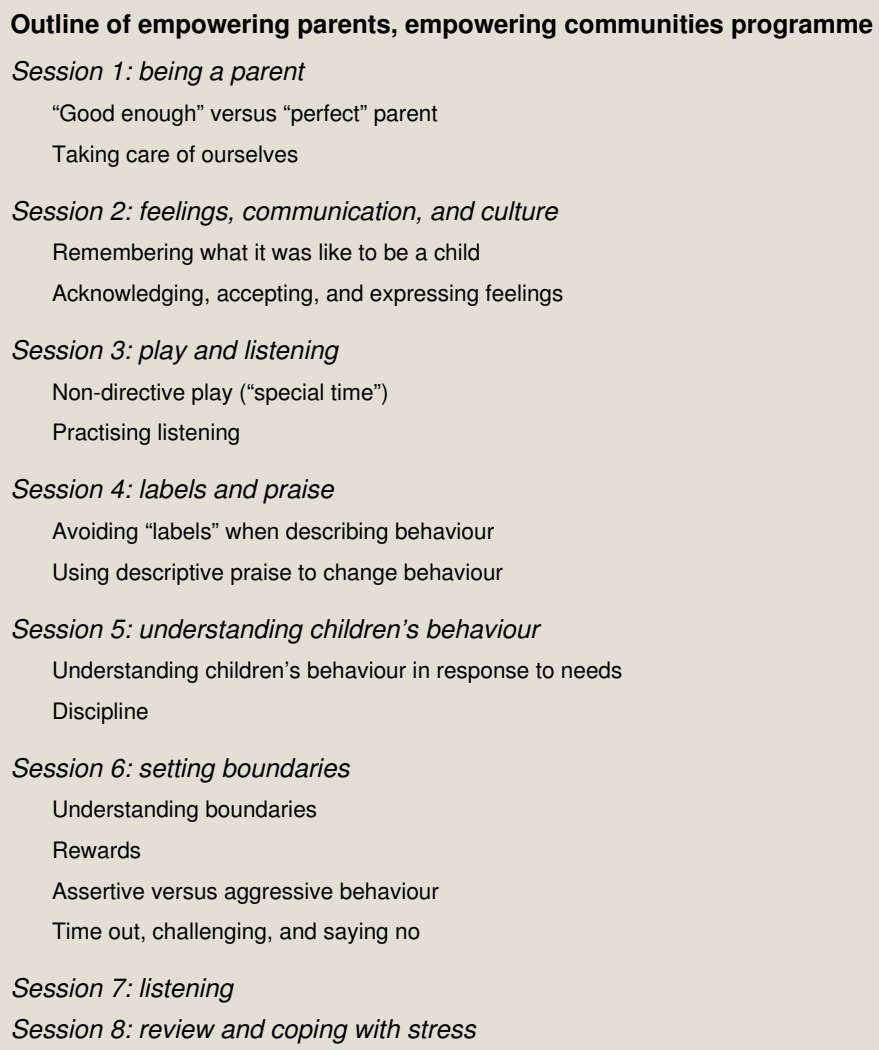

variation in the type and number of nominated problems, we limited the analysis of severity ratings to specified "number one" concerns.

The strengths and difficulties questionnaire ${ }^{22}$ is a 25 item screening measure used to obtain a more global assessment of child mental health problems. It produces a total difficulties score and four problem subscale scores (emotional problems, conduct problems, hyperactivity/inattention, peer relationship problems), with higher scores indicating more dysfunction.

The Arnold-O'Leary parenting scale ${ }^{23}$ is a 30 item scale that assesses parenting competencies, providing a total score and three subscales (laxness, over-reactivity, and verbosity). Lower scores indicate more adaptive parenting strategies.

The parenting stress index-short form ${ }^{24}$ is a 36 item questionnaire that assesses parental adjustment across three subscales (parental distress, difficult child, and parent-child dysfunctional interaction). Higher scores indicate greater levels of stress in the parenting role.

\section{User acceptability}

The treatment acceptability rating scale ${ }^{25}$ was used in an adapted form ${ }^{15}$ to assess the perceived treatment utility and quality of experience for parents who participated in the peer led parenting groups. The measure obtains ratings of user satisfaction for nine different aspects of treatment (using a four point Likert scale), and also elicits qualitative feedback from three open ended questions.

\section{Sample size}

The sample size calculation was based on a previous randomised controlled trial ${ }^{26}$ carried out in community settings with socially disadvantaged families, in which parent training groups (facilitated by professionals) were compared with a waitlist control condition. An intention to treat analysis showed an intervention effect size of 0.89 (95\% confidence interval 0.54 to 1.24 ) and a mean difference of 25.1 (95\% confidence interval 14.9 to $35.2, \mathrm{P}<0.001$ ) on the intensity subscale of the Eyberg child behaviour inventory. Taking the lower bound of this confidence interval as a conservative estimate of effectiveness, we calculated that 110 families would be needed to detect a significant effect $(\mathrm{P}<0.05)$ of parent training in the present study.

\section{Randomisation}

We randomised participants by intervention site in blocks of between 7 and 14, depending on the number of families recruited at a given site. If two adults from the same family wished to attend the programme, we included both in the same unit of randomisation along with the index child. Otherwise the unit of randomisation was the index child-parent pair.

An independent trial technician used computer software to oversee the randomisation procedure. A 1:1 allocation ratio was used so that equal numbers of participants were assigned to the intervention and waitlist control groups. Allocation took place after baseline assessment; blinding of post-intervention assessments was not possible owing to the use of parent reported outcome measures.

\section{Analysis strategy}

We used an intention to treat strategy-that is, all participants were included in the analysis irrespective of whether they had completed the intervention. For participants who were lost to follow-up we assumed no change from baseline. The results for a per protocol analysis were also calculated. Analysis of covariance was used to test differences between intervention and waitlist control groups at follow-up, accounting for site as a random effect, intervention as a fixed effect, and baseline score as a covariate. We used Cohen's d to calculate effect sizes. 


\section{Results}

The figure $\Downarrow$ shows the flow of recruited families through the trial. Table $1 \Downarrow$ shows the baseline characteristics of the families, including those lost to follow-up. A large proportion of parents identified themselves as being from black and ethnic minority groups $(n=82 ; 71 \%)$, double the proportion of residents from black and ethnic minority groups in Southwark as a whole. ${ }^{27}$ The study participants were also more economically disadvantaged than the general population of Southwark across a range of indices, including income and housing status. ${ }^{28}$

\section{Uptake of intervention}

Fifty four parents $(92 \%)$ completed the parenting intervention, defined as attendance at five or more (out of a total of eight) weekly sessions. Those completing the intervention attended a mean 7.10 (SD 0.92) sessions. Among the five non-completers, two did not attend any sessions, one attended one session, one attended two sessions, and one attended four sessions. Reasons for non-attendance were new employment $(n=1)$, travel overseas to care for a relative $(n=1)$, lack of readiness to engage $(n=1)$, and unknown $(\mathrm{n}=2)$.

\section{Main findings}

The intervention group showed significant improvements on all outcome measures after treatment $(\mathrm{P}<0.01$ or $\mathrm{P}<0.001)$. In contrast, no outcome measure changed significantly within the control group. This pattern extended to levels of caseness within the respective groups (table $2 \Downarrow$ ).

Intention to treat analyses of differences between the groups showed significant advantages for the intervention group over control group on the intensity and problems subscales of the Eyberg child behaviour inventory $(\mathrm{P}=0.01$ and $\mathrm{P}=0.001)$ and concerns about my child $(\mathrm{P}<0.001)$, with medium to large effect sizes of between 0.38 and 0.77 on these measures of child behaviour problems (table $3 \Downarrow$ ). The groups did not differ significantly on overall child mental health functioning (strengths and difficulties questionnaire total score) or on any of the individual subscales of the strengths and difficulties questionnaire. The intervention had a significant, moderately large effect on positive parenting (Arnold-O'Leary parenting scale total score) compared with the control group $(\mathrm{P}<0.001$; $\mathrm{d}=0.69$, table $4 \Downarrow$ ). However, parental stress (parenting stress index-short form total score) did not differ significantly between the groups. This pattern of results was replicated in the per protocol analyses (see tables 3 and 4).

Fifty four parents in the intervention group completed the training acceptability rating scale at the end of treatment. High levels of treatment acceptability were reported, with $100 \%$ of respondents stating that overall they were satisfied "a great deal" or "quite a lot." Specific aspects of the intervention were also strongly endorsed, with a median acceptability score of 4 (the maximum possible) for every item on the training acceptability rating scale. This included ratings of the perceived competence of the facilitators (mean 3.87, SD 0.34), ability of the facilitators to relate to the group (mean $3.79,0.41$ ), motivational qualities of the facilitators (mean 3.83, SD 0.38), as well as the extent to which the intervention led to increased understanding of positive parenting (mean 3.59, SD 0.53), development of positive parenting skills (mean 3.50, SD 0.64), and confidence in the parenting role (mean 3.44, SD 0.69).

\section{Discussion}

A peer led parenting intervention can significantly reduce behaviour problems in children and improve positive parenting in families characterised by a high degree of socioeconomic disadvantage. Moreover, the low rate of drop out suggests that a peer led approach is an acceptable means of delivering evidence based parenting support to families who may not otherwise engage in mainstream services. Although parental stress significantly decreased in the intervention group, the advantage of treatment over a waitlist control condition on this particular outcome was not significant.

The peer led parenting intervention achieved medium to large effects on both the primary outcome measure (Eyberg child behaviour inventory-intensity subscale) and secondary outcome measures of child behaviour problems and parenting competencies. Effect sizes compared favourably with results from clinical trials in which parenting interventions were delivered in a conventional professional led format. ${ }^{626}$ Previous trials $^{29}{ }^{30}$ have also tended to report relatively larger intervention effects on child behaviour outcomes compared with parental adjustment, as was the case in the present study. It is therefore likely that our trial was underpowered to detect a significant advantage for the intervention over the control group in reducing parental stress.

In terms of engagement outcomes, the intervention had an impressively high retention rate of $92 \%$. This finding is particularly notable given the sociodemographic profile of the participating parents, which would typically be associated with multiple barriers to service use. ${ }^{89}{ }^{10}$ In comparison, retention rates of $75 \%$ and $85 \%{ }^{26}$ have been reported in two other UK trials of professional led parenting programmes for socially disadvantaged families. This suggests that the current peer led parenting intervention has the potential to outperform conventional parenting group formats, even those specifically targeted at hard to reach populations, in successfully engaging parents in treatment for their children's behaviour problems.

\section{Strengths and limitations of the study}

The present study is a novel evaluation of the effectiveness of a peer led parenting intervention, delivered in a socially deprived inner city area of the United Kingdom. The trial was carried out in community settings as a part of the routine provision of the empowering parents, empowering communities programme, which strengthens the external validity of findings and generalisability to conditions in the real world. Randomisation and allocation concealment were employed to minimise selection bias and maximise internal validity. However, we cannot rule out the possibility of measurement bias due to unblinded follow-up assessments, or shared method bias owing to assessment based entirely on parental self report measures. Future research should employ independent observations of interactions between parent and child, or reports from other key informants such as teachers. As yet, follow-up data to determine the longer term effects of the empowering parents, empowering communities programme are lacking, although the results suggest that such research may be warranted.

\section{Meanings and implications of the study}

This study has shown that a peer led parenting intervention can provide accessible and effective treatment for disruptive behaviour problems in children from some of the most vulnerable families in society. The results are of particular interest in the current sociopolitical climate, where services are subject to intense budgetary pressures and there is urgent need 
for innovative, cost effective delivery models. Although formal economic evaluations have yet to be completed, the intervention may offer an economically viable method for increasing provision of evidence based parenting support for families who are hard to reach.

\section{Questions and future research}

Our findings require replication for children with different levels of problem severity, in comparison with other active treatments, such as treatment as usual or another standardised parenting programme, and in longer term follow-up studies. In addition, future studies should collect more comprehensive information on the baseline characteristics of participating families, such as the mental health status of parents, to examine the variation in treatment response across different sociodemographic and clinical profiles.

Research is also needed to examine the cost effectiveness of the empowering parents, empowering communities programme, as well as the wider effects of the intervention on social capital and community resources. This would be of importance not only to the further development of the intervention but more broadly to the understanding of peer led approaches and their potential benefits for the health and wellbeing of individuals, families, and communities.

We thank Caroline Adewole, Annika Jaramillo, and Catherine Kearney for their assistance on the empowering parents, empowering communities project; the peer facilitators and parents involved with the project; Stephen Rock and Susan Tilki for their assistance with data collection; and Guy's and St Thomas' Charity and the London Borough of Southwark for funding.

Contributors: $C D$ was principal investigator, obtained funding, and acts as guarantor for the research. DM and CD designed the study. ST carried out consent and randomisation procedures; collected, managed, and analysed data; and wrote the first draft of the paper under the supervision of DM. DM and CD critically revised the manuscript. CP and LD advised on the manuscript, authored the intervention manual, and supervised the peer facilitators.

Funding: This study was supported by Guy's and St Thomas' Charity and the London Borough of Southwark.

Competing interests: All authors have completed the ICMJE uniform disclosure form at www.icmje.org/coi_disclosure.pdf (available on request from the corresponding author) and declare that: all authors have support from the Institute of Psychiatry, King's College London for the submitted work; all authors have no relationships with any organisations that might have an interest in the submitted work in the previous three years; and all authors have no non-financial interests that may be relevant to the submitted work.

Ethical approval. This study was approved by the National Health Service National Research Ethics Service (South West 2 research ethics committee).

Data sharing: No additional data available.

1 Barlow J, Underdown A. Promoting the social and emotional health of children: where to now? J R Soc Prom Health 2005;125:64-70.
2 Burke JD, Loeber R, Birmaher B. Oppositional defiant disorder and conduct disorder: a review of the past 10 years, part II. J Am Acad Child Adolesc Psychiatry 2002:41:1275-93.

3 Loeber R, Farrington DP. Young children who commit crime: epidemiology, developmental origins, risk factors, early interventions, and policy implications. Dev Psychopathol 2000;12:737-62.

4 Davis H, Day C, Cox A, Cutler L. Child and adolescent mental health needs assessment and service implications in an inner city area. Clin Child Psychol Psychiat 2000;5:168-9.

5 Fergusson DM, Horwood LJ, Ridder EM. Show me the child at seven: the consequences of conduct problems in childhood for psychosocial functioning in adulthood. J Child Psychol Psychiatry 2005;46:837-49.

6 Dretzke J, Davenport C, Frew E, Barlow J, Stewart-Brown S, Bayliss S, et al. The clinical effectiveness of different parenting programmes for children with conduct problems: a systematic review of randomised controlled trials. Clin Adolesc Psychiatry Ment Health 2009;3:289-99.

7 National Institute for Health and Clinical Excellence. Parent-training/education programmes in the management of children with conduct disorders. NICE, 2006

8 Nock MK, Ferriter C. Parent management of attendance and adherence in child and adolescent therapy: a conceptual and empirical review. Clin Child Fam Psychol Rev 2005;8:149-66.

9 McKay MM, Hoagwood K, Murray L, Fernandez D. Integrating evidence-based engagement interventions in "real world" child mental health settings. Brief Treat Crisis Interv 2004:4:177-86.

10 Forehand R, Kotchick BA. Cultural diversity: a wake-up call for parent training. Behav Ther 1996;27:187-206.

11 Kazdin AE, Holland L, Crowley M. Family experience of barriers to treatment and premature termination from child therapy. J Consul Clin Psychol 1997;65:453-63.

12 Cupples ME, Stewart MC, Percy A, Hepper P, Murphy C, Halliday HL. An RCT of peer-mentoring for first-time mothers in socially disadvantaged areas (the MOMENTS Study). Arch Dis Child 2010;96:252-8

13 Konrad SC. What parents of seriously ill children value: parent-to-parent connection and mentorship. Omega (Westport) 2007:55:117-30.

14 Webel AR, Okonsky J, Trompeta J, Holzemer WL. A systematic review of the effectiveness of peer-based interventions on health-related behaviors in adults. Am J Public Health 2010;100:247-53.

15 Day C, Michelson D, Thomson S, Penney C, Draper L. Empowering parents, empowering communities: a pilot evaluation of a peer-led parenting programme. Child Adolesc Ment Health 2012;17:52-7.

16 Schulz KF, Altman DG, Moher D. CONSORT 2010 statement: updated guidelines for reporting parallel group randomised trials. BMJ 2010;340:c332.

17 Penney C, Wilson C, Draper L, Day C, Kearney C. Being a parent: manual for course facilitators. The Parenting Centre and the Centre for Parent and Child Support, 2010.

18 Eyberg S, Ross AW. Assessment of child behaviour problems: the validation of a new inventory. J Clin Child Psychol 1978;7:113-6.

19 Rich BA, Eyberg S. Accuracy of assessment: the discriminative and predictive power of the Eyberg Child Behaviour Inventory. Am J Child Health 2001:7:249-57.

20 Eyberg SM, Pincus D. The Eyberg Child Behavior Inventory and the Sutter-Eyberg Student Behaviour Inventory-Revised: professional manual. Psychological Assessment Resources, 1999.

21 Scott S, Spender Q, Doolan M, Jacobs B, Aspland H. Multicentre controlled trial of parenting groups for childhood antisocial behaviour in clinical practice. $B M J$ 2001:323:194-6.

22 Goodman R. The Strengths and Difficulties Questionnaire: a research note. J Child Psychol Psychiatry 1997;38:581-6.

23 Arnold DS, O'Leary SG, Wolff LS, Acker M. The Parenting Scale: a measure of dysfunctional parenting in discipline situations. Psychol Assess 1993;5:137-44.

24 Abidin RR. Parenting Stress Index Professional Manual. 3rd ed. Psychological Assessment Resources, 1995.

25 Davis JR, Rawana EP, Copponi DR. Acceptability of behavioural staff management techniques. Behav Res Treat 1989;4:32-44.

26 Hutchings J, Bywater T, Daley D, Gardner F, Whitaker C, Jones K, et al. Parenting intervention in Sure Start services for children at risk of developing conduct disorder: pragmatic randomised controlled trial. BMJ 2007;334:678-82.

27 Southwark Analytical Hub. Southwark vital statistics 2008. London Borough of Southwark, 2009.

28 Southwark Analytical Hub. English indices of deprivation 2007. London Borough of Southwark, 2008

29 Serketich WJ, Dumas JE. The effectiveness of behavioural parent training to modify antisocial behaviour in children: a meta-analysis. Behav Ther 1996;27:171-86.

30 Barlow J, Coren E, Stewart-Brown S. Meta-analysis of the effectiveness of parenting programmes in improving maternal psychosocial health. Br J Gen Pract 2002;52:223-33.

Accepted: 22 December 2011

\section{Cite this as: BMJ 2012;344:e1107}

This is an open-access article distributed under the terms of the Creative Commons Attribution Non-commercial License, which permits use, distribution, and reproduction in any medium, provided the original work is properly cited, the use is non commercial and is otherwise in compliance with the license. See: http://creativecommons.org/licenses/by$\mathrm{nc} / 2.0 /$ and http://creativecommons.org/licenses/by-nc/2.0/legalcode. 


\section{What is already known on this topic}

Parenting interventions are recommended for the treatment of disruptive behaviour problems in children aged up to 12 years However, standard parenting interventions, as delivered by professionals in mainstream services, are often inaccessible to the most vulnerable families

\section{What this study adds}

A peer led parenting intervention can significantly reduce behaviour problems in children and improve positive parenting in socioeconomically disadvantaged families

The $<10 \%$ dropout rate suggests that a peer led group format is an acceptable method for delivering evidence based parenting support to families who may not otherwise engage in mainstream services

\section{Tables}

\begin{tabular}{|c|c|c|c|c|c|}
\hline \multirow[b]{2}{*}{ Characteristics } & \multicolumn{2}{|c|}{ Followed-up } & \multicolumn{2}{|c|}{ Lost to follow-up } & \multirow[b]{2}{*}{ Total sample $(n=116)$} \\
\hline & Control $(n=50)$ & Intervention $(n=54)$ & Control $(n=7)$ & Intervention $(n=5)$ & \\
\hline Boys & $34(68)$ & $32(59)$ & $2(29)$ & $1(20)$ & 69 (59) \\
\hline Mean (SD) age of child (years) & $4.82(2.35)$ & $4.70(2.47)$ & $5.00(2.71)$ & $5.80(3.77)$ & $4.81(2.46)$ \\
\hline Biological mothers & $48(96)$ & $51(94)$ & $7(100)$ & $5(100)$ & $111(96)$ \\
\hline Mean (SD) age of parent (years) & $35.82(7.76)$ & $37.02(7.60)$ & $33.00(4.08)$ & $33.40(4.93)$ & $36.10(7.44)$ \\
\hline Parent from black and ethnic minority group & $38(76)$ & $37(69)$ & $5(71)$ & $2(40)$ & $82(71)$ \\
\hline Disposable weekly household income $<£ 200^{*}$ & $34(68)$ & $36(67)$ & $5(71)$ & $3(50)$ & $78(67)$ \\
\hline \multicolumn{6}{|l|}{ Housing status: } \\
\hline Rent from local authority & $19(38)$ & $19(35)$ & $2(29)$ & $2(40)$ & $42(36)$ \\
\hline Rent privately & $13(26)$ & $14(26)$ & $3(43)$ & $1(20)$ & $31(27)$ \\
\hline Rent from housing association & $8(16)$ & $4(7)$ & $1(14)$ & - & $13(11)$ \\
\hline Owner occupiers & $2(4)$ & $12(22)$ & $1(14)$ & - & $15(13)$ \\
\hline Other/missing & $8(16)$ & $5(9)$ & - & $2(40)$ & $15(13)$ \\
\hline
\end{tabular}




\begin{tabular}{|c|c|c|c|c|c|c|}
\hline \multirow[b]{2}{*}{ Measure } & \multicolumn{3}{|c|}{ Control group } & \multicolumn{3}{|c|}{ Intervention group } \\
\hline & No* & Baseline & Follow-upt & No* & Baseline & Follow-upt \\
\hline \multicolumn{7}{|l|}{ Eyberg child behaviour inventory: } \\
\hline $\begin{array}{l}\text { Intensity subscale (clinical range } \\
\geq 127 \text { ) }\end{array}$ & 57 & $17(30)$ & $18(32)$ & 58 & $24(41)$ & $11(19)$ \\
\hline $\begin{array}{l}\text { Problems subscale (clinical range } \\
\geq 11 \text { ) }\end{array}$ & 52 & $25(48)$ & $21(40)$ & 54 & $24(44)$ & $9(17)$ \\
\hline \multicolumn{7}{|l|}{$\begin{array}{l}\text { Strengths and difficulties } \\
\text { questionnaire: }\end{array}$} \\
\hline $\begin{array}{l}\text { Total (abnormal/borderline range } \\
\geq 14 \text { ) }\end{array}$ & 47 & $14(30)$ & $16(34)$ & 45 & $17(38)$ & $10(22)$ \\
\hline $\begin{array}{l}\text { Conduct (abnormal/borderline range } \\
\geq 3 \text { ) }\end{array}$ & 47 & $17(36)$ & $17(36)$ & 46 & $22(48)$ & $15(33)$ \\
\hline $\begin{array}{l}\text { Hyperactivity/inattention } \\
\text { (abnormal/borderline range } \geq 6 \text { ) }\end{array}$ & 47 & $16(34)$ & $12(26)$ & 45 & $14(31)$ & $11(24)$ \\
\hline Parenting scale (clinical range $\geq 3.1$ ) & 56 & $31(55)$ & $36(64)$ & 59 & $39(66)$ & $23(39)$ \\
\hline $\begin{array}{l}\text { Parenting stress index-short form } \\
\text { (clinical range } \geq 90 \text { ) }\end{array}$ & 57 & $19(33)$ & $19(33)$ & 59 & $31(53)$ & $14(24)$ \\
\hline
\end{tabular}

*Variation in number is due to failure of some participants to complete all questionnaires during data collection meetings; number is lowest for strengths and difficulties questionnaire because scores for 2 year olds were excluded (measure is valid for children aged 3 years and over).

tWhen follow-up score was missing, baseline measurement was carried forward as per intention to treat analysis. 


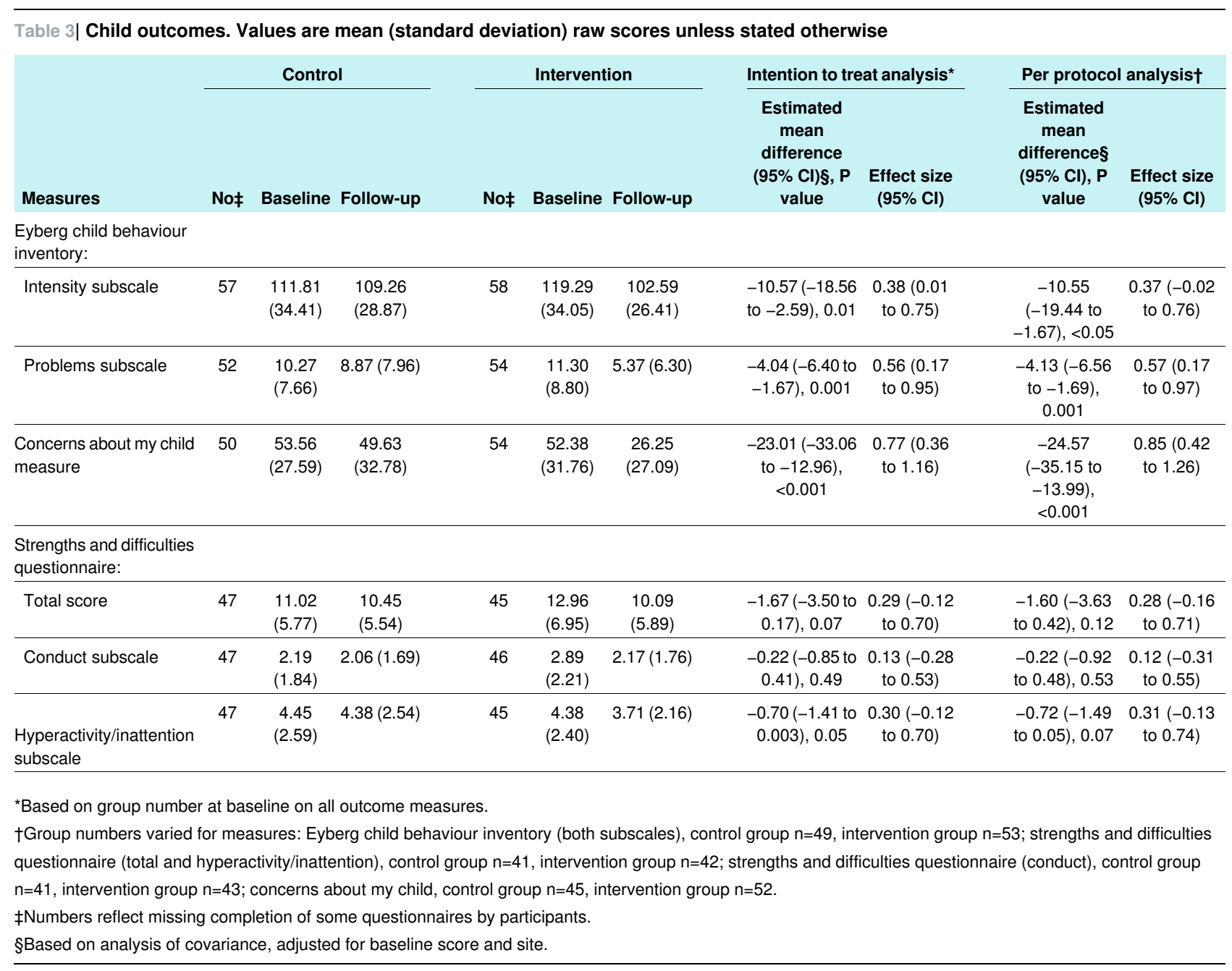


Table 4| Parenting outcomes. Values are mean (standard deviation) raw scores unless stated otherwise

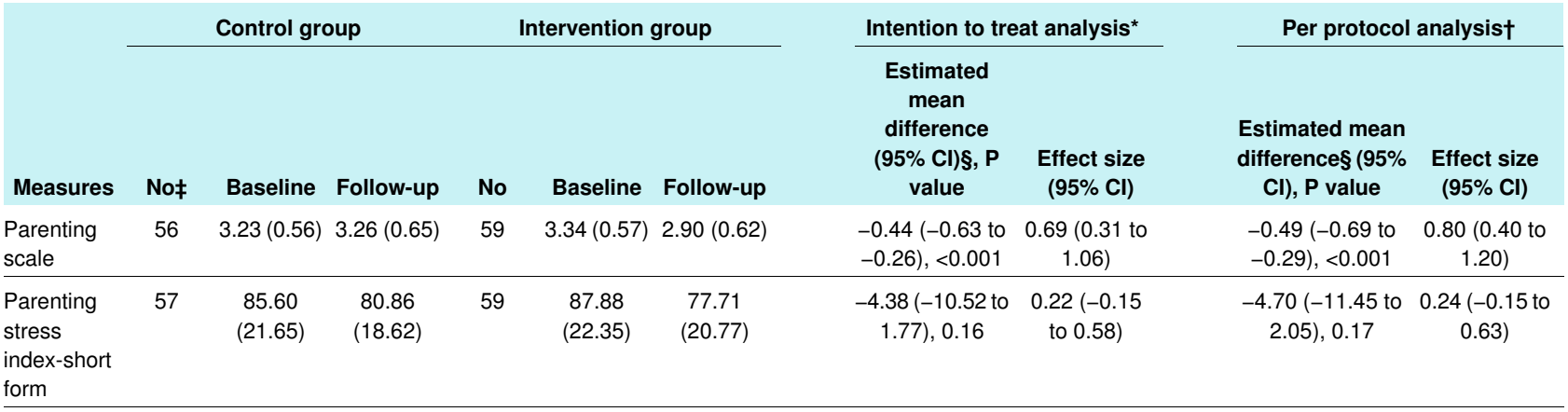

*Based on group number at baseline on all outcome measures.

†Group numbers varied for measures: parenting scale, control group $n=50$, intervention group $n=54$; parenting stress index-short form, control group $n=48$,

intervention group $\mathrm{n}=54$.

¥Numbers reflect missing completion of some questionnaires by participants.

$\S$ Based on analysis of covariance, adjusted for baseline score and site. 


\section{Figure}

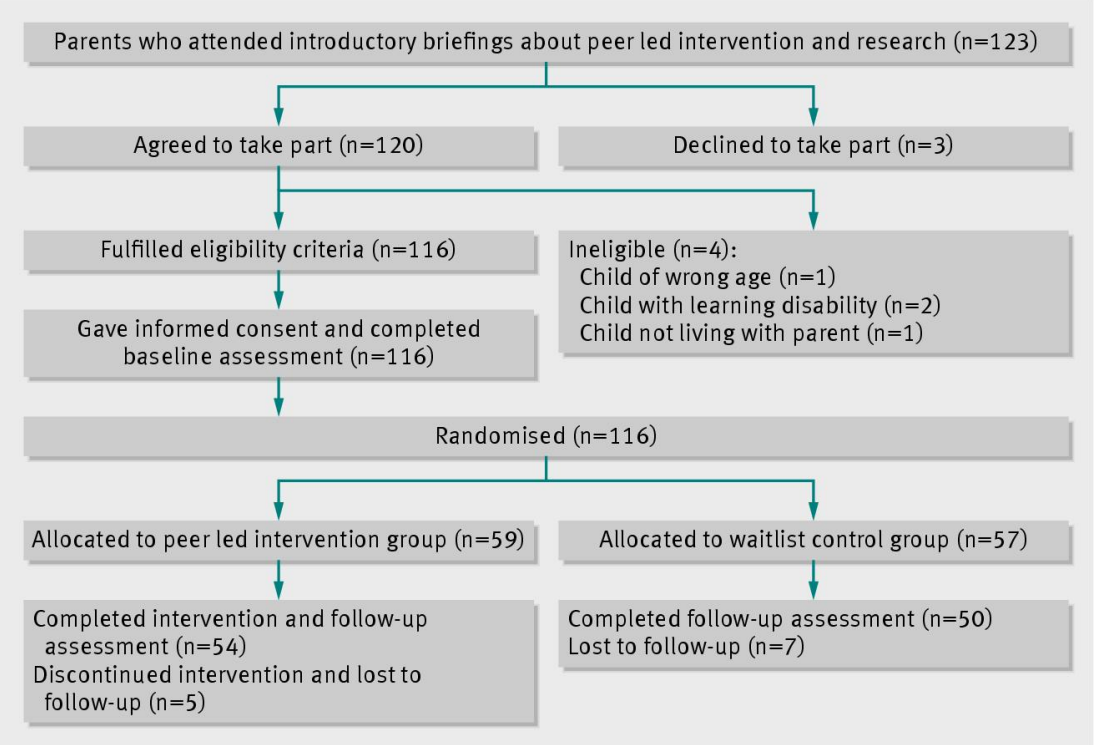

Flow of families through trial 